\title{
Long noncoding RNA PXN-AS1-L promotes non-small cell lung cancer progression via regulating PXN
}

Zhifa Zhang ${ }^{1 \dagger}$, Zhaohui Peng ${ }^{2,3 \dagger}$, Junying Cao ${ }^{4 \dagger}$, Jiaqi Wang ${ }^{5}$, Yongyu Hao ${ }^{1}$, Kai Song ${ }^{1}$, Yan Wang ${ }^{1}$, Wei Hu ${ }^{6 *}$ and Xuesong Zhang ${ }^{1 *}$ (D)

\begin{abstract}
Background: Increasingly evidences suggest that long noncoding RNAs (IncRNAs) play important roles in various cancers. LncRNA PXN-AS1-L is recently revealed to act as on oncogene in liver cancer. However, the expression, functions, and mechanisms of action of PXN-AS-L in non-small cell lung cancer (NSCLC) remain unclear.

Methods: The expression of PXN-AS1-L in primary NSCLC tissues, NSCLC bone metastasis tissues, and cell lines was measured by quantitative real-time PCR. The correlations between PXN-AS1-L expression and clinicopathological characteristics of NSCLC patients were analyzed by Pearson Chi square test and log-rank test. The roles of PXN-AS1-L in cell viability, proliferation, apoptosis, and migration of NSCLC cells, and in vivo NSCLC tumor growth were investigated by a series of gain-of-function and loss-of-function assays. The regulatory roles of PXN-AS1-L on PXN were determined by quantitative real-time PCR and western blot.

Results: PXN-AS1-L was up-regulated in NSCLC tissues compared with noncancerous lung tissues, and PXN-AS1L was further up-regulated in NSCLC bone metastasis tissues. Increased expression of PXN-AS1-L was positively associated with advanced TNM stages and poor prognosis. Gain-of-function and loss-of-function assays showed that PXN-AS1-L increased cell viability, promoted cell proliferation, inhibited cell apoptosis, and promoted cell migration of NSCLC cells. Xenograft assays showed that PXN-AS1-L also promoted NSCLC tumor growth in vivo. Mechanistically, we found that PXN-AS1-L, as an antisense transcript of PXN, up-regulated the expression of PXN. PXN was also up-regulated in NSCLC tissues. The expression of PXN and PXN-AS1-L was positively correlated in NSCLC tissues. Furthermore, PXN knockdown attenuated the roles of PXN-AS1-L in increasing cell viability, promoting cell proliferation, inhibiting cell apoptosis, and promoting cell migration of NSCLC cells.
\end{abstract}

Conclusions: Our data revealed that PXN-AS1-L is up-regulated and acts as an oncogene in NSCLC via up-regulating PXN. Our data suggested that PXN-AS1-L might serve as a potential prognostic biomarker and therapeutic target for NSCLC.

Keywords: Long noncoding RNA, Non-small-cell lung cancer, Proliferation, Migration, PXN

\footnotetext{
*Correspondence: huweicj@163.com; zhangxuesong301@sina.com

†Zhifa Zhang, Zhaohui Peng and Junying Cao contributed equally to this work

1 Department of Orthopaedic Surgery, the PLA General Hospital,

Beijing 100000, China

${ }^{6}$ Department of General Surgery, Changhai Hospital, Second Military

Medical University, Shanghai 200433, China

Full list of author information is available at the end of the article
}

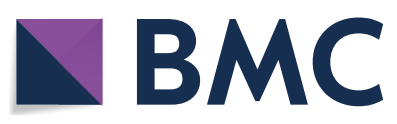

(c) The Author(s) 2019. This article is distributed under the terms of the Creative Commons Attribution 4.0 International License (http://creativecommons.org/licenses/by/4.0/), which permits unrestricted use, distribution, and reproduction in any medium, provided you give appropriate credit to the original author(s) and the source, provide a link to the Creative Commons license, and indicate if changes were made. The Creative Commons Public Domain Dedication waiver (http://creativecommons.org/ publicdomain/zero/1.0/) applies to the data made available in this article, unless otherwise stated. 


\section{Background}

Lung cancer is the most common malignancy and the leading cause of cancer death around the world, with an estimated 2,093,876 new cases and 1,761,007 deaths in 2018 worldwide [1]. The major histological subtype of lung cancer is non-small cell lung cancer (NSCLC), which mainly includes adenocarcinoma and squamous cell carcinoma, and accounts for approximate $80 \%$ of lung cancers [2]. Except the NSCLC patients which are diagnosed at early stages and could be cured by surgical resection, most NSCLC patients still have poor outcomes with the 5-year survival rate of about $20 \%$ [3]. Thus, it is of paramount importance to reveal the underlying molecular mechanisms contributing to the tumorigenesis and progression of NSCLC in order to develop novel therapeutic strategies for NSCLC [4-6].

Accumulating evidences have revealed that most of human genome is transcribed, but only about $2 \%$ of human genome encode for proteins [7]. Therefore, most of human transcripts don't encode for proteins. Among these non-coding transcripts, long noncoding RNAs (lncRNAs) have gradually attracted people's attention [8-12]. LncRNA is a class of non-coding transcripts with more than 200 nucleotides in length and limited protein coding potential [13-15]. Many lncRNAs are revealed to play important roles in various pathophysiological processes [16-19]. As to tumors, lncRNAs have been reported to regulate almost every aspect of biological behaviors of cancer cells, including cell proliferation, apoptosis, senescence, autophagy, migration, invasion, and so on [20-25]. Furthermore, many lncRNAs are dysregulated in various cancers and associated with early diagnosis and/or prognosis [26-28].

Several IncRNAs have been reported to have oncogenic or tumor suppressing roles in NSCLC. LncRNA MALAT1 is a positive marker of lung cancer metastasis [29]. LncRNA PVT1 is reported to promote NSCLC cell proliferation [30]. LncRNA BANCR is reported to promote NSCLC metastasis [31]. LncRNA MIR22HG is reported to inhibit cell survival [32]. LncRNA P53RRA is reported to promote ferroptosis and apoptosis [33]. LncRNA MUC5B-AS1 is reported to promote metastasis [34]. LncRNA linc00460 is reported to promote cell migration [35]. LncRNA LINC00473 is reported to promote lung cancer tumor growth [36]. Although the expression and function of these lncRNAs are studied in NSCLC, most lncRNAs transcribed from human genome are functionally unclear in NSCLC [37]. LncRNA PXN-AS1-L is a recently identified lncRNA, which is upregulated in hepatocellular carcinoma (HCC) and promoted HCC tumorigenesis via up-regulating PXN [38]. However, the expression, roles, and mechanisms of action of lncRNA PXN-AS1-L in NSCLC are still unknown.
In this study, we aimed to elucidate the expression pattern of lncRNA PXN-AS1-L in NSCLC via measuring PXN-AS1-L expression in noncancerous lung tissues, NSCLC tissues, NSCLC bone metastases tissues, normal bronchial epithelial cell line, and human NSCLC cell lines. We also investigated the biological functions of PXN-AS1-L in NSCLC using in vitro and in vivo gainof-function and loss-of-function assays. Moreover, we explored the molecular mechanisms mediating the roles of PXN-AS1-L in NSCLC.

\section{Methods}

\section{Cell culture and treatment}

The human normal bronchial epithelial cell line 16HBE, human NSCLC cell lines NCI-H1975, A549, NCI-H1299, and SK-MES-1 were obtained from Cell Bank of Type Culture Collection of the Chinese Academy of Sciences (Shanghai, China). 16HBE cells were cultured in Dulbecco's Modified Eagle's Medium (Invitrogen, Carlsbad, CA, USA). NCI-H1975 and NCI-H1299 cells were cultured in RPMI-1640 Medium (Invitrogen). A549 cells were cultured in F-12K Medium (Invitrogen). SK-MES-1 cells were cultured in Eagle's Minimum Essential Medium (Invitrogen). All the cells were maintained in the above described medium supplemented with $10 \%$ fetal bovine serum (Invitrogen) in a humidified incubator at $37{ }^{\circ} \mathrm{C}$ with $5 \% \mathrm{CO}_{2}$. Where indicated, NSCLC cells were treated with $50 \mu \mathrm{M} \alpha$-amanitin (Sigma-Aldrich, Saint Louis, MO, USA) for $0-24 \mathrm{~h}$ as shown in the article.

\section{Human tissue specimens}

Sixty-six pairs of NSCLC tissues and adjacent noncancerous lung tissues, and ten NSCLC bone metastases tissues were acquired from NSCLC patients who underwent surgery at the General Hospital of Chinese People's Liberation Army (Beijing, China). All the tissues were diagnosed and histologically confirmed by two pathologists. The resected specimens were immediately frozen in liquid nitrogen and stored at $-80{ }^{\circ} \mathrm{C}$ until use. The study was approved by the ethics committee of the General Hospital of Chinese People's Liberation Army (Beijing, China) and written informed consent was obtained from all patients.

\section{Plasmids construction and transfection}

PXN-AS1-L overexpression plasmid pcDNA3.1PXN-AS1-L was constructed as previously described [38]. Briefly, PXN-AS1-L full-length transcript was PCR amplified by Thermo Scientific Phusion Flash High-Fidelity PCR Master Mix (Thermo-Fisher Scientific, Waltham, MA, USA) and subcloned into the BamH I and EcoR V sites of pcDNA3.1 plasmid (Invitrogen). The primers sequences were: 5'-GGTACCGAGCTCGGATCCTCG 
CGTTGGAGGAGCTTG-3' (forward) and 5'-GCCACT GTGCTGGATATCCTACAAAAAAAATTTATTTAA TAAAA- $3^{\prime}$ (reverse). The cDNA oligonucleotides suppressing PXN-AS1-L or PXN expression were designed and synthesized by GenePharma (Shanghai, China). After annealing, double strand oligonucleotides were inserted to the SuperSilencing shRNA expression plasmid pGPU6/Neo (GenePharma). A scrambled shRNA was used as a negative control and designated as shControl. The shRNA sequences were: for shPXN, 5'-CAC CCCTGACGAAAGAGAAGCCTAATTCAAGAGATT AGGCTTCTCTTTCGTCAGGTTTTTTG-3' (sense), $5^{\prime}$-GATCCAAAAAACCTGACGAAAGAGAAGCCT AATCTCTTGAATTAGGCTTCTCTTTCGTCAGG-3' (anti-sense); for shRXN-AS1-L, 5'-CACCGCCCAGAG GAAATCAACAAGATTCAAGAGATCTTGTTGATT TCCTCTGGGCTTTTTTG-3' (sense), 5'-GATCCA AAAAAGCCCAGAGGAAATCAACAAGATCTCTTG AATCTTGTTGATTTCCTCTGGGC-3' (anti-sense); for shControl, 5'-CACCGTTCTCCGAACGTGTCA CGTTTCAAGAGAACGTGACACGTTCGGAGAATT TTTTG-3' (sense), 5'-GATCCAAAAAATTCTCCGAAC GTGTCACGTTCTCTTGAAACGTGACACGTTCGG AGAAC-3' (anti-sense). Transient transfection was performed using Lipofectamine 3000 (Invitrogen) in accordance with the manufacturer's instruction.

\section{Construction of stable cell lines}

To constructing PXN-AS1-L stably overexpressed A549 cells, pcDNA3.1-PXN-AS1-L or pcDNA3.1 was transfected into A549 cells and selected with neomycin $(800 \mu \mathrm{g} / \mathrm{ml})$ for 4 weeks. To constructing PXN-AS1-L stably depleted NCI-H1299 cells, shPXN-AS1-L or shControl was transfected into NCI-H1299 cells and selected with neomycin $(1000 \mu \mathrm{g} / \mathrm{ml})$ for 4 weeks.

\section{RNA extraction and quantitative real-time PCR analysis (qPCR)}

Total RNA was extracted from indicted tissues or cells using TRIzol Reagent (Invitrogen) in accordance with the manufacturer's protocol. Reverse transcription was performed using the M-MLV Reverse Transcriptase (Invitrogen) in accordance with the manufacturer's protocol. Quantitative real-time PCR (qPCR) was performed in the StepOnePlus Real-Time PCR System (Applied Biosystems, Foster City, CA, USA) using the SYBR ${ }^{\circledR}$ Premix Ex Taq $^{\mathrm{TM}}$ II kit (Takara, Dalian, China) in accordance with the manufacturer's protocols. $\beta$-actin was used as an endogenous control. The primers sequences were: for PXN-AS1-L, 5'-ACCCATCCTCAACTACCCC-3' (forward) and $5^{\prime}$-ACTTCGTCTGTGCCTTCTGC- $3^{\prime}$ (reverse); for PXN, 5'-TATCTCAGCCCTCAACAC GC-3' (forward) and 5'-GGCAGAAGGCACAGACGA
A-3' (reverse); for $\beta$-actin, $5^{\prime}$-GGGAAATCGTGCGTG ACATTAAG-3' (forward) and 5'-TGTGTTGGCGTA CAGGTCTTTG-3' (reverse). The relative expression of RNAs was calculated using the comparative $\mathrm{Ct}$ method.

\section{Western blot analysis}

Total proteins were extracted using RIPA buffer (Beyotime, Shanghai, China). Identical quantities of proteins were separated by sodium dodecyl sulfate-polyacrylamide gel electrophoresis and transferred onto nitrocellulose filter membranes. After being blocked with 5\% not-fat milk, the membranes were incubated with primary antibodies specific for PXN (Abcam, Hong Kong, China) or $\beta$-actin (Proteintech, Rosemont, IL, USA). After being washed, the membranes were incubated with IRdye 700-conjugated goat anti-mouse IgG or IRdye 800-conjugated goat anti-rabbit IgG and were detected using an Odyssey infrared scanner (Li-Cor, Lincoln, NE, USA).

\section{Analysis of cell proliferation and apoptosis}

Cell proliferation was detected using Glo cell viability assay and Ethynyl deoxyuridine (EdU) incorporation assay. For Glo cell viability assay, 3000 indicated NSCLC cells were seeded each well in 96-well plate. At indicated time, cell viability was detected using the CellTiter-Glo Luminescent Cell Viability Assay (Promega, Madison, WI, USA) in accordance with the manufacturer's protocol. EdU incorporation assay was carried out using the EdU kit (Roche, Mannheim, Germany) in accordance with the manufacturer's protocol. The results were acquired and quantified with the Zeiss AxioPhot Photomicroscope (Carl Zeiss, Oberkochen, Germany) based on at least ten random fields. Cell apoptosis was detected using terminal deoxynucleotidyl transferase (TdT)-mediated dUTP nick end labeling (TUNEL) assay. Indicated NSCLC cells were treated with $25 \mathrm{ng} / \mathrm{ml}$ doxorubicin (Selleck, Houston, TX, USA) for $24 \mathrm{~h}$. Then, cell apoptosis was detected using the TUNEL Cell Apoptosis Detection Kit (Beyotime) in accordance with the manufacturer's protocol.

\section{Cell migration assay}

Cell migration was detected using transwell assay. $5 \times 10^{4}$ indicated NSCLC cells in serum-free medium were plated in the top chamber a 24-well transwell chamber (BD Biosciences, San Jose, CA, USA). Complete medium containing $10 \%$ fetal bovine serum was placed into the lower chamber. After incubation for $24 \mathrm{~h}$, cells remaining on the top chamber were wiped off using a cotton swab, and the cells that had traversed the membranes were stained by crystal violet and counted. 

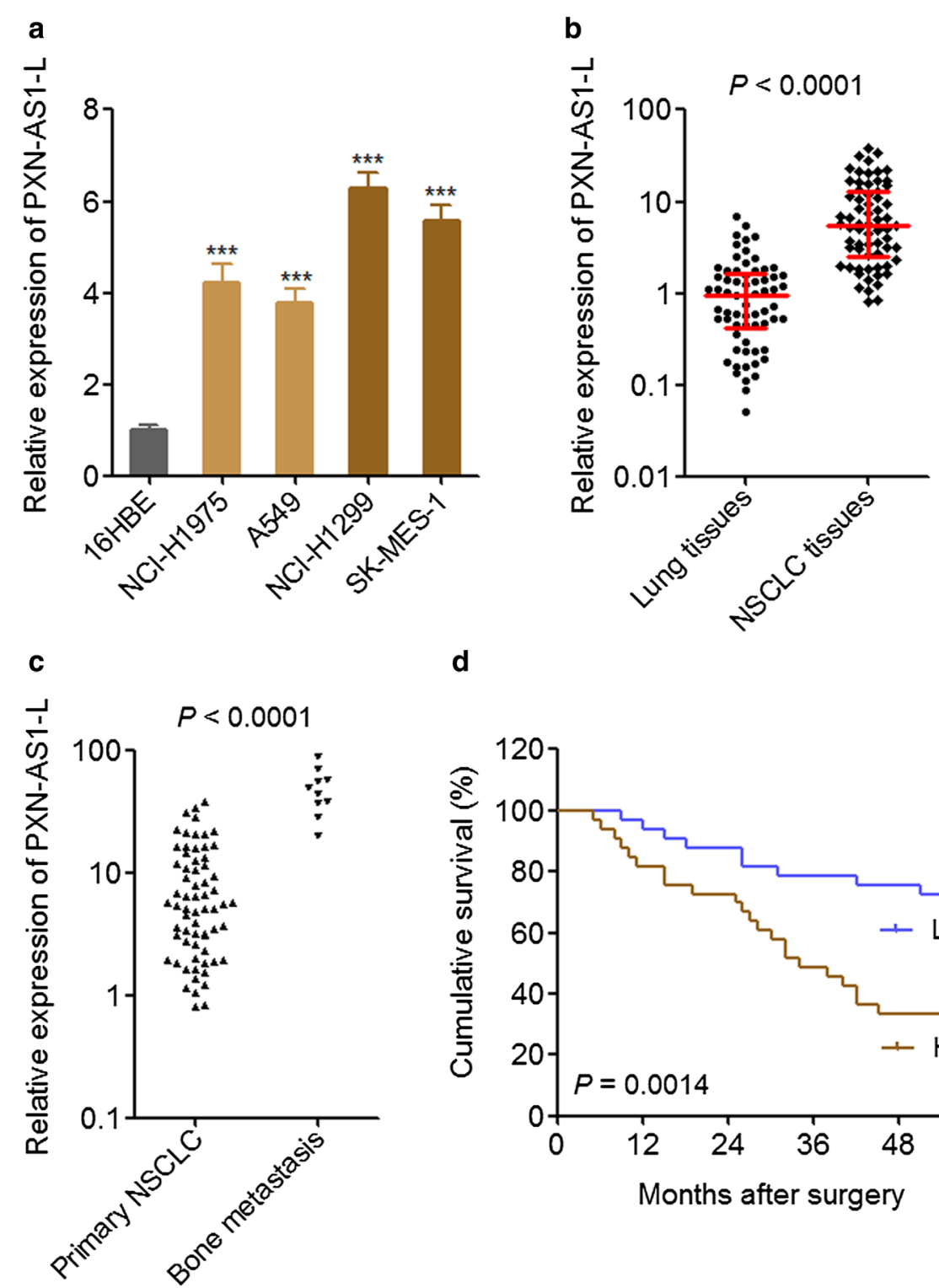

d

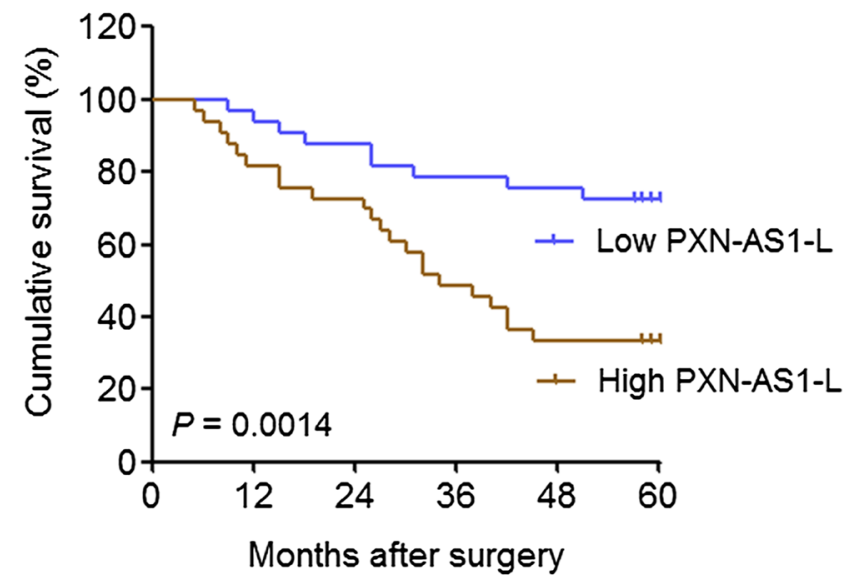

Fig. 1 PXN-AS1-L was up-regulated in NSCLC and associated with poor prognosis. a The expressions of PXN-AS1-L in normal bronchial epithelial cell line 16HBE and NSCLC cell lines NCI-H1975, A549, NCI-H1299, and SK-MES-1 were detected by qPCR. Results are shown as mean \pm SD of three independent experiments. ${ }^{* *} P<0.001$ by Student's $t$-test. $\mathbf{b}$ The expressions of PXN-AS1-L in 66 pairs of NSCLC tissues and adjacent noncancerous lung tissues were detected by qPCR. $P<0.0001$ by Wilcoxon signed-rank test. $\mathbf{c}$ The expressions of PXN-AS1-L in 66 primary NSCLC tissues and 10 NSCLC bone metastases tissues were detected by qPCR. $P<0.0001$ by Mann-Whitney test. $\mathbf{d}$ Kaplan-Meier survival analysis of the correlation between PXN-AS1-L expression and overall survival of these 66 NSCLC patients. The median PXN-AS1-L expression level was used as the cut-off. $P<0.0001$ by Log-rank test

\section{Nude mouse xenograft assays}

$3 \times 10^{6}$ indicted NSCLC cells were subcutaneously injected into 6-week-old athymic BALB/c nude mice purchased from the Shanghai Experimental Animal Center of Chinese Academy of Sciences (Shanghai, China). Subcutaneous xenograft growth was measured weekly with a caliper, and the tumor volume was calculated as $\mathrm{a} \times \mathrm{b}^{2} \times 0.5$ (a, longest diameter; $\mathrm{b}$, shortest diameter).
The animal studies were approved by the ethics committee of the General Hospital of Chinese People's Liberation Army (Beijing, China).

\section{Immunohistochemistry (IHC)}

For IHC, the subcutaneous xenografts were formalinfixed, paraffin-embedded, and cut into $4 \mu \mathrm{m}$ sections. The 
Table 1 Correlation between PXN-AS1-L expression and clinicopathological characteristics of NSCLC patients

\begin{tabular}{|c|c|c|c|c|}
\hline \multirow[t]{2}{*}{ Variables } & \multirow[t]{2}{*}{ Number } & \multicolumn{2}{|c|}{ PXN-AS1-L } & \multirow[t]{2}{*}{$P$ value* } \\
\hline & & Low & High $^{a}$ & \\
\hline All cases & 66 & 33 & 33 & \\
\hline Age & & & & 0.620 \\
\hline$\geq 60$ & 37 & 20 & 17 & \\
\hline$<60$ & 29 & 13 & 16 & \\
\hline Gender & & & & 1.000 \\
\hline Male & 48 & 24 & 24 & \\
\hline Female & 18 & 9 & 9 & \\
\hline Histologic subtype & & & & 1.000 \\
\hline Squamous cell carcinoma & 30 & 15 & 15 & \\
\hline Adenocarcinoma & 36 & 18 & 18 & \\
\hline Tumor size $(\mathrm{cm})$ & & & & 0.021 \\
\hline$\leq 3$ & 11 & 9 & 2 & \\
\hline$>3$ & 55 & 24 & 31 & \\
\hline Lymph nodes metastasis & & & & 0.003 \\
\hline Positive & 26 & 7 & 19 & \\
\hline Negative & 40 & 26 & 14 & \\
\hline TNM stage & & & & 0.000 \\
\hline । & 28 & 23 & 5 & \\
\hline$\|$ & 27 & 10 & 17 & \\
\hline III & 11 & 0 & 11 & \\
\hline
\end{tabular}

a The median expression level of PXN-AS1-L was used as the cut-off * $P$ value was acquired by Pearson Chi square test

sections were incubated with primary antibodies specific for Ki67 (Abcam) or cleaved caspase-3 (Cell Signaling Technology, Boston, MA, USA). After being washed, the sections were incubated with horseradish peroxidaseconjugated second antibody (Abcam) and visualized using DAB Horseradish Peroxidase Color Development Kit (Beyotime).

\section{Statistical analysis}

All statistical analyses were performed using the GraphPad Prism Software. For comparisons, Student's $t$ test (two-sided), Wilcoxon signed-rank test, Mann-Whitney test, Pearson Chi square test, Log-rank test, and Pearson correlation analysis were performed as indicated. $P$ values $<0.05$ were considered as statistically significant.

\section{Results}

PXN-AS1-L was up-regulated in NSCLC and associated with poor prognosis

To investigate the expression pattern of PXN-AS1-L in NSCLC, we first measured the expression of PXN-AS1$\mathrm{L}$ in normal bronchial epithelial cell line $16 \mathrm{HBE}$ and
NSCLC cell lines NCI-H1975, A549, NCI-H1299, SKMES-1. The results displayed that PXN-AS1-L was significantly up-regulated in NSCLC cell lines compared with that in normal bronchial epithelial cell line, and further up-regulated in NSCLC cell lines derived from metastatic sites (NCI-H1299 and SK-MES-1) (Fig. 1a). Then, we collected 66 pairs of NSCLC tissues and adjacent noncancerous lung tissues and measured the expression of PXN-AS1-L in these tissues. The results displayed that the expression of PXN-AS1-L was significantly higher in NSCLC tissues than that in adjacent noncancerous lung tissues (Fig. 1b). Furthermore, we collected 10 NSCLC bone metastases tissues and also measured the expression of PXN-AS1-L. The results displayed that the expression of PXN-AS1-L was further higher in bone metastases tissues than that in primary NSCLC tissues (Fig. 1c).

The correlations between the expression of PXN-AS1$\mathrm{L}$ in NSCLC tissues and clinicopathological characteristics of these 66 NSCLC patients were analyzed. As shown in Table 1, increased expression of PXN-AS1-L was positively associated with larger tumor size, positive lymph nodes metastasis, and advanced TNM stages, but not associated with age, gender, and histologic subtype. Kaplan-Meier survival analysis displayed that NSCLC patients with higher PXN-AS1-L expression had worse survival than those with lower PXN-AS1-L expression (Fig. 1d). All these data together demonstrated that PXN-AS1-L was up-regulated in NSCLC, further up-regulated in metastatic NSCLC cells and tissues. Increased expression of PXN-AS1-L predicted poor outcome of NSCLC patients.

\section{PXN-AS1-L overexpression promoted NSCLC cell proliferation and migration}

To reveal the biological effects of PXN-AS1-L on NSCLC, we stably overexpressed PXN-AS1-L in A549 cells which has a relative low expression of PXN-AS1$\mathrm{L}$ among NSCLC cell lines by transfecting PXN-AS1-L overexpression plasmid (Fig. 2a). Glo cell viability assays displayed that PXN-AS1-L overexpression increased cell viability of A549 cells (Fig. 2b). EdU incorporation assays also displayed that PXN-AS1-L overexpression promoted cell proliferation of A549 cells (Fig. 2c). TUNEL assays displayed that PXN-AS1-L overexpression inhibited cell apoptosis of A549 cells (Fig. 2d). Transwell assays displayed that PXN-AS1-L overexpression promoted cell migration of A549 cells (Fig. 2e). All these data together demonstrated that PXN-AS1-L overexpression promoted cell proliferation, inhibited cell apoptosis, and promoted cell migration of NSCLC cells, suggesting that PXN-AS1$\mathrm{L}$ has oncogenic roles in NSCLC. 


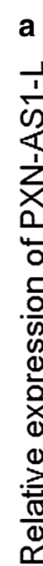

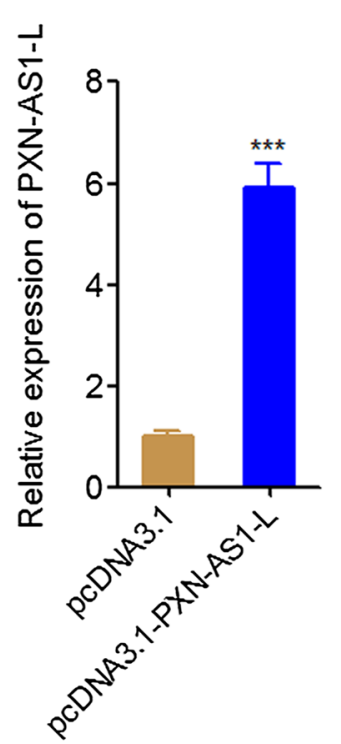

b

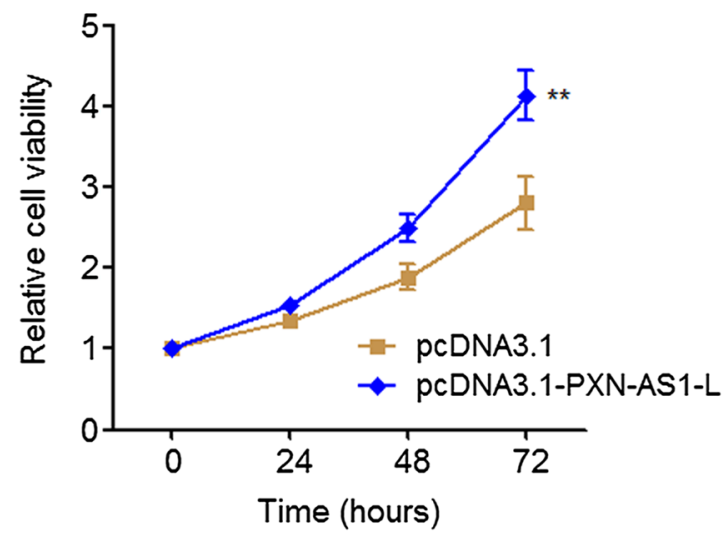

C

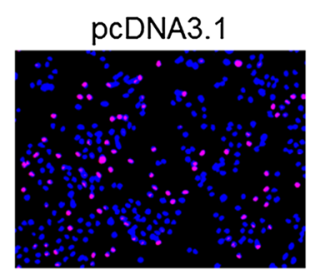

pcDNA3.1-PXN-AS1-L
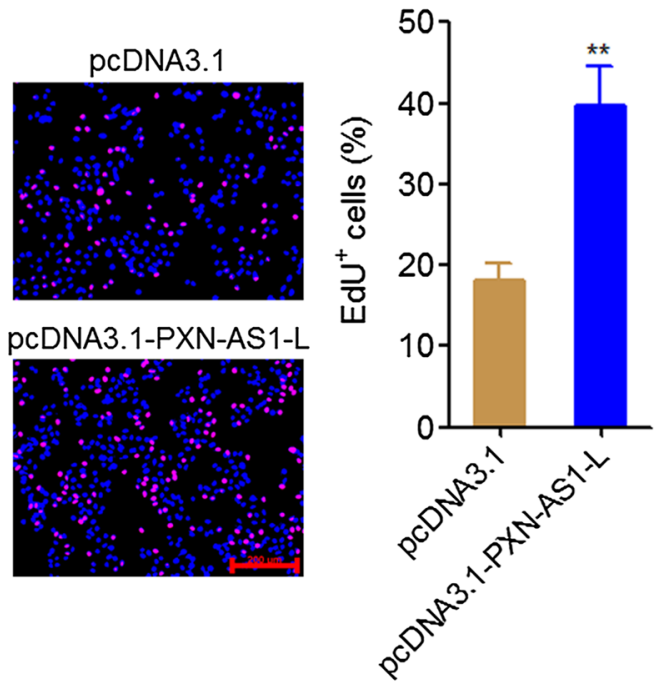

d

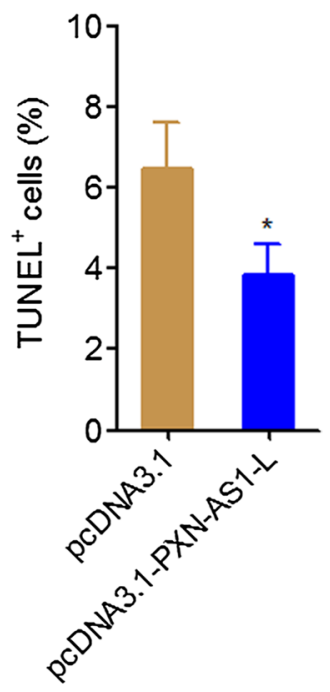

e
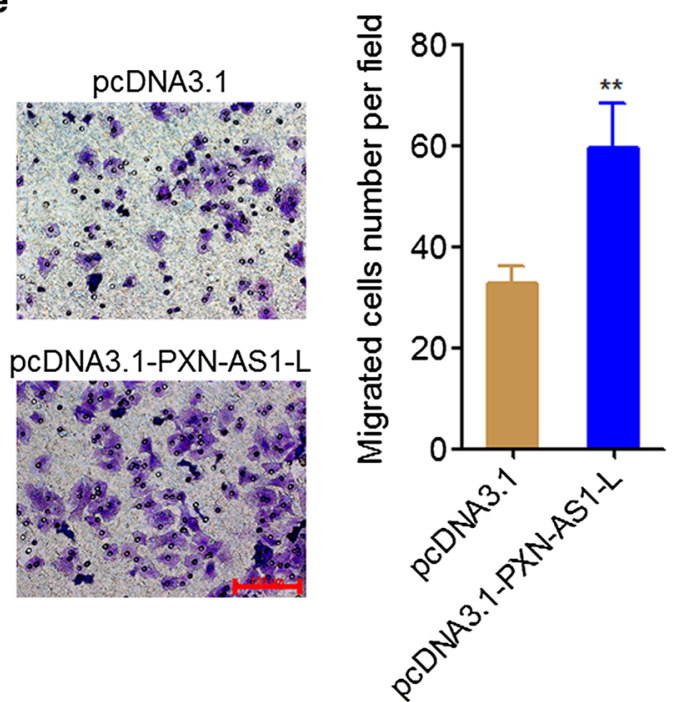

Fig. 2 PXN-AS1-L overexpression promoted NSCLC cell proliferation and migration. a The expressions of PXN-AS1-L in PXN-AS1-L stably overexpressed and control A549 cells were detected by qPCR. b Cell viability of PXN-AS1-L stably overexpressed and control A549 cells was detected by Glo cell viability assays. c Cell proliferation of PXN-AS1-L stably overexpressed and control A549 cells was detected by EdU incorporation assays. The red color indicates EdU-positive cells. Scale bars $=200 \mu \mathrm{m}$. d Cell apoptosis of PXN-AS1-L stably overexpressed and control A549 cells was detected by TUNEL assays. e Cell migration of PXN-AS1-L stably overexpressed and control A549 cells was detected by transwell assays. Scale bars $=100 \mu \mathrm{m}$. Results are shown as mean \pm SD of three independent experiments. ${ }^{*} P<0.05,{ }^{* *} P<0.01,{ }^{* *} P<0.001$ by Student's $t$-test

\section{PXN-AS1-L knockdown reduced NSCLC cell proliferation} and migration

To further confirm the oncogenic roles of PXN-AS1L on NSCLC, we stably knocked down PXN-AS1-L expression in NCI-H1299 cells which has a relative high expression of PXN-AS1-L among NSCLC cell lines by transfecting PXN-AS1-L specific shRNA (Fig. 3a).
Glo cell viability assays displayed that PXN-AS1-L knockdown reduced cell viability of NCI-H1299 cells (Fig. 3b). EdU incorporation assays also displayed that PXN-AS1-L knockdown inhibited cell proliferation of NCI-H1299 cells (Fig. 3c). TUNEL assays displayed that PXN-AS1-L knockdown promoted cell apoptosis of NCI-H1299 cells (Fig. 3d). Transwell assays displayed 


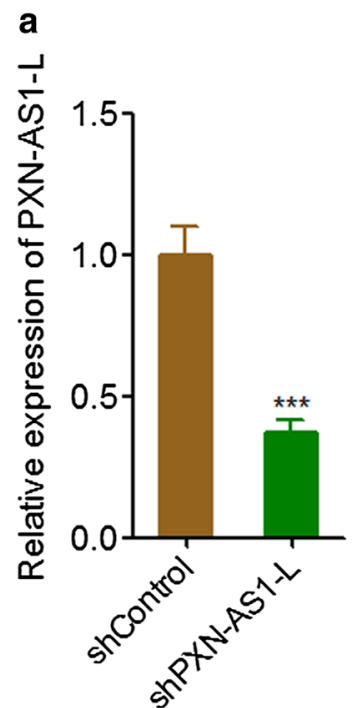

C

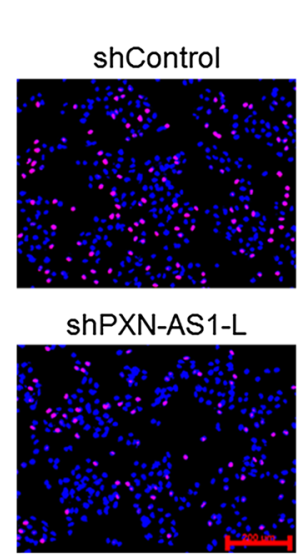

b

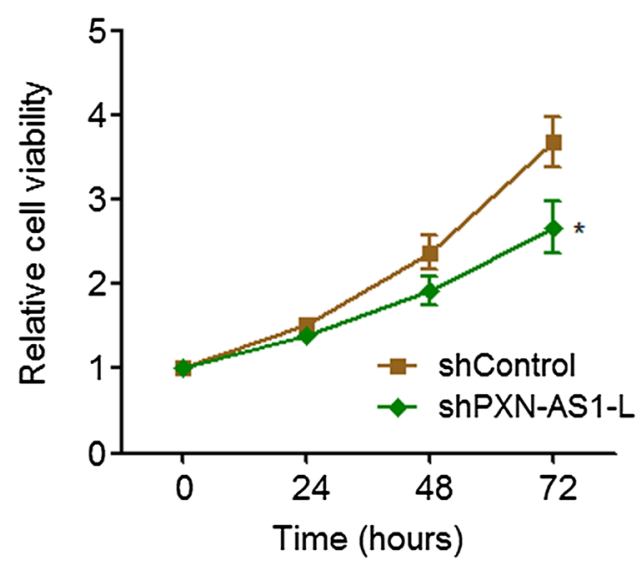

e d

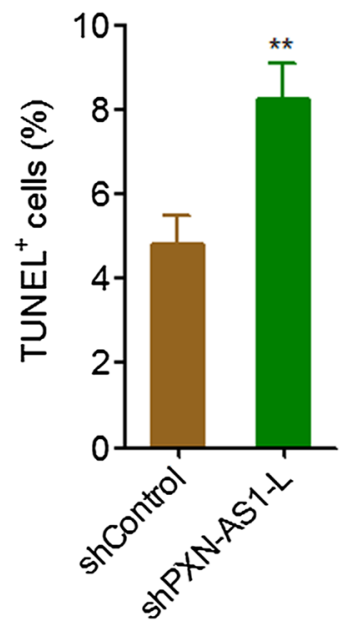

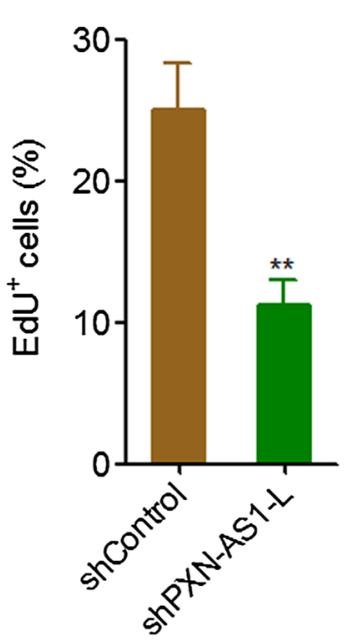
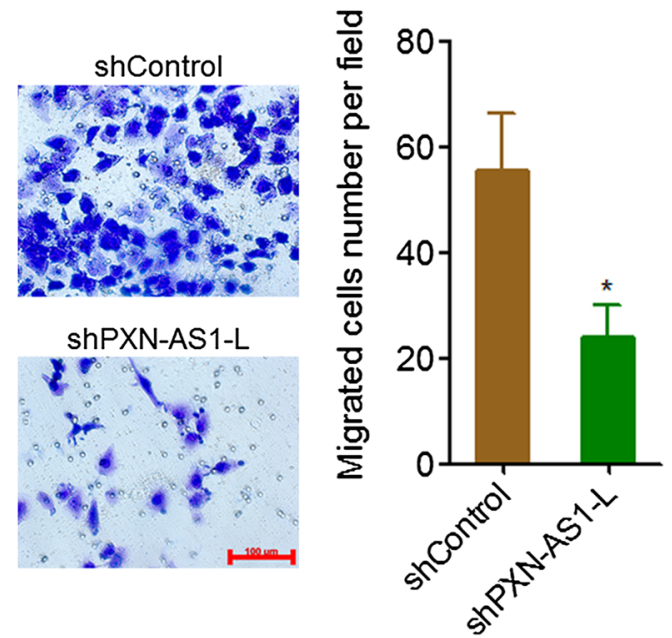

Fig. 3 PXN-AS1-L knockdown inhibited NSCLC cell proliferation and migration. a The expressions of PXN-AS1-L in PXN-AS1-L stably knocked down and control NCI-H1299 cells were detected by qPCR. b Cell viability of PXN-AS1-L stably knocked down and control NCI-H1299 cells was detected by Glo cell viability assays. c Cell proliferation of PXN-AS1-L stably knocked down and control NCI-H1299 cells was detected by EdU incorporation assays. The red color indicates EdU-positive cells. Scale bars $=200 \mu \mathrm{m}$. d Cell apoptosis of PXN-AS1-L stably knocked down and control NCI-H1299 cells was detected by TUNEL assays. e Cell migration of PXN-AS1-L stably knocked down and control NCI-H1299 cells was detected by transwell assays. Scale bars $=100 \mu \mathrm{m}$. Results are shown as mean \pm SD of three independent experiments. ${ }^{*} P<0.05,{ }^{* *} P<0.01,{ }^{* * *} P<0.001$ by Student's $t$-test

that PXN-AS1-L knockdown inhibited cell migration of NCI-H1299 cells (Fig. 3e). All these data together demonstrated that PXN-AS1-L knockdown inhibited cell proliferation, promoted cell apoptosis, and inhibited cell migration of NSCLC cells, supporting the oncogenic roles of PXN-AS1-L in NSCLC.

\section{PXN-AS1-L promoted the growth of NSCLC xenograft in vivo}

To explore whether PXN-AS1-L also have oncogenic roles in vivo, PXN-AS1-L stably overexpressed and control A549 cells were subcutaneously injected into nude mice. Xenografts growth rates were measured every 7 days. At the 28th day, the xenografts were excised and weighed. The results displayed that PXN-AS1-L overexpression markedly promoted xenograft growth in vivo, and PXN-AS1-L overexpressed A549 cells formed much larger tumor than those formed by control A549 cells (Fig. 4a, b). Ki67 staining of xenograft displayed that PXN-AS1-L overexpression promoted A549 cell proliferation in vivo (Fig. 4c). Cleaved caspase-3 staining of xenograft displayed that PXN-AS1-L overexpression 


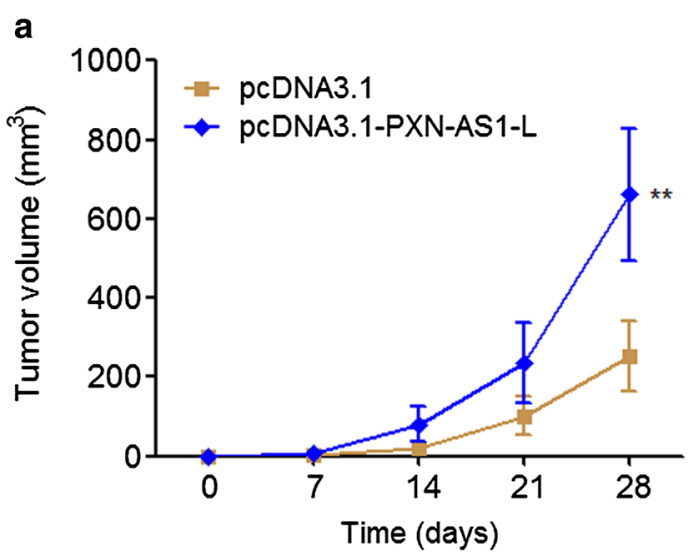

c
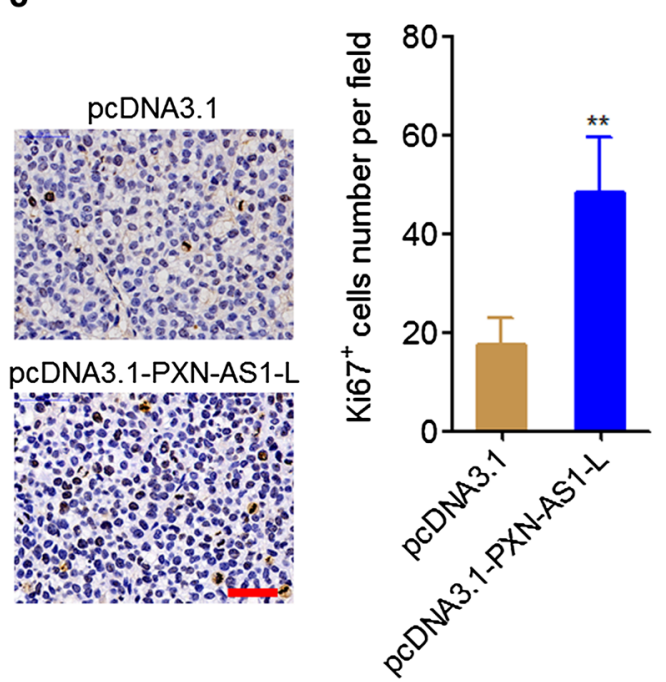

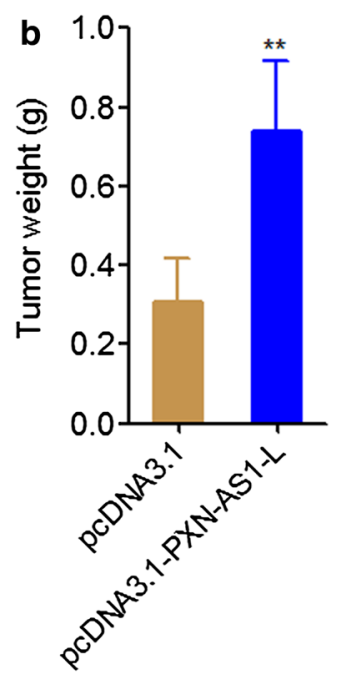

d

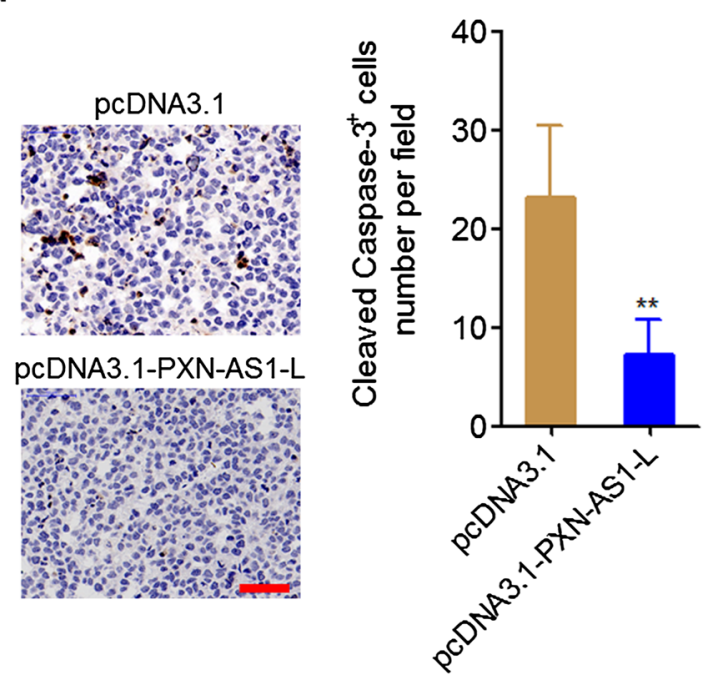

Fig. 4 PXN-AS1-L promoted NSCLC xenograft growth in vivo. a Tumor volumes of subcutaneous xenografts derived from PXN-AS1-L stably overexpressed and control A549 cells were measured every 7 days. b Tumor weights of subcutaneous xenografts derived from PXN-AS1-L stably overexpressed and control A549 cells at the 28th day after injection. c In vivo cell proliferation of PXN-AS1-L stably overexpressed and control A549 cells was evaluated using Ki67 immunohistochemistry staining of subcutaneous xenografts. Scale bars $=50 \mu \mathrm{m}$. $\mathbf{d}$ In vivo cell apoptosis of PXN-AS1-L stably overexpressed and control A549 cells was evaluated using cleaved caspase-3 immunohistochemistry staining of subcutaneous xenografts. Scale bars $=50 \mu \mathrm{m}$. Results are shown as mean \pm SD of six mice in each group. ${ }^{* *} P<0.01$ by Mann-Whitney test

inhibited A549 cell apoptosis in vivo (Fig. 4d). All these data together demonstrated that PXN-AS1-L also had oncogenic roles in vivo.

\section{PXN-AS1-L up-regulated PXN expression}

PXN-AS1-L is previously reported to up-regulate PXN expression in HCC cells [38]. PXN has been revealed to play oncogenic roles in NSCLC in several reports [3941]. Therefore, we first investigated whether PXN-AS1-L also regulates PXN expression in NSCLC and whether the oncogenic roles of PXN-AS1-L in NSCLC are dependent on the regulation of PXN. The expressions of PXN in PXN-AS1-L stably overexpressed and control A549 cells, and PXN-AS1-L stably depleted and control NCI-H1299 cells were measured by qRT-PCR and western blot. The results displayed that PXN-AS1-L overexpression up-regulated PXN mRNA level (Fig. 5a). Conversely, PXN-AS1-L knockdown reduced PXN mRNA level (Fig. 5b). Western blot assays also displayed that PXN-AS1-L overexpression up-regulated PXN protein level and while PXN-AS1-L knockdown reduced PXN protein level (Fig. 5c, d). PXN-AS1-L is reported 


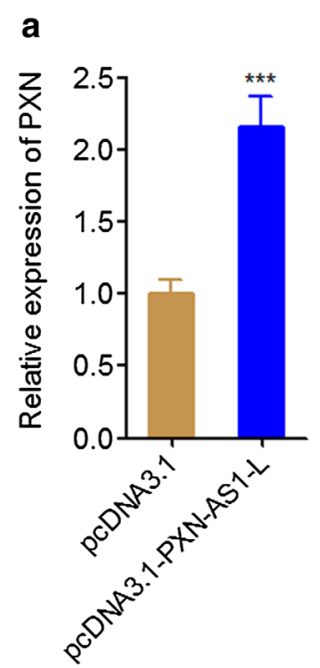

e

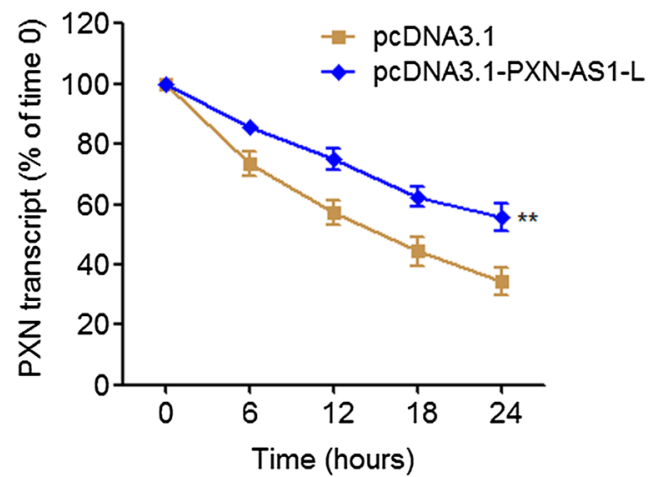

C

d
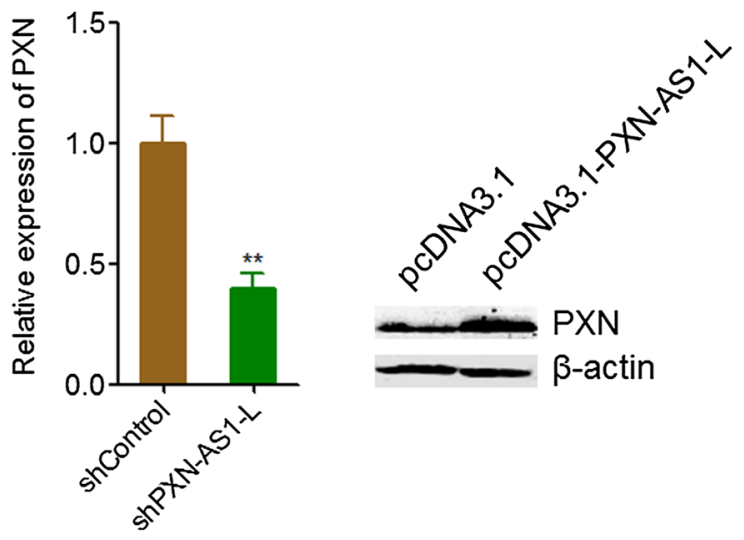

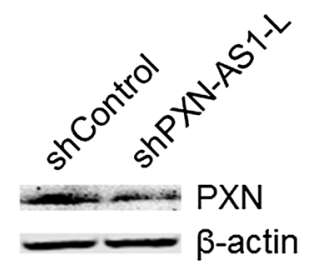

$\mathbf{f}$

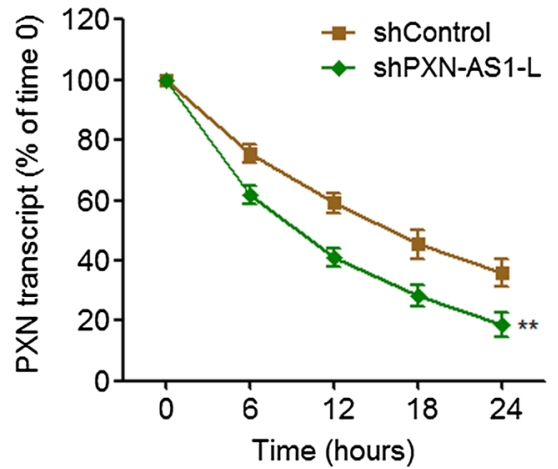

Fig. 5 PXN-AS1-L up-regulated PXN expression. a PXN mRNA levels in PXN-AS1-L stably overexpressed and control A549 cells were detected by qPCR. b PXN mRNA levels in PXN-AS1-L stably depleted and control NCl-H1299 cells were detected by qPCR. c PXN protein levels in PXN-AS1-L stably overexpressed and control A549 cells were detected by western blot. d PXN protein levels in PXN-AS1-L stably depleted and control $\mathrm{NCl}-\mathrm{H} 1299$ cells were detected by western blot. e After transient overexpressing PXN-AS1-L in A549 cells, the stability of PXN mRNA over time was determined after blocking new RNA synthesis with a-amanitin $(50 \mu \mathrm{M})$ and normalized to $18 \mathrm{~S}$ rRNA (a product of RNA polymerase I that is unchanged by a-amanitin). f After transient depleting PXN-AS1-L in NCl-H1299 cells, the stability of PXN mRNA over time was determined after blocking new RNA synthesis with a-amanitin $(50 \mu \mathrm{M})$ and normalized to $18 \mathrm{~S} r R N A$. Results are shown as mean \pm SD of three independent experiments. ${ }^{* *} P<0.01,{ }^{* * *} P<0.001$ by Student's $t$-test

to decrease PXN mRNA degradation and increase PXN mRNA stability in HCC cells [38]. To investigate whether the same mechanism was employed by PXN-AS1-L in NSCLC, we next determined the effects of PXN-AS1-L on PXN mRNA stability in NSCLC cell. After transiently overexpressing PXN-AS1-L in A549 cells or depleting PXN-AS1-L in NCI-H1299 cells, the cells were treated with $\alpha$-amanitin to block new RNA synthesis. Next, the loss of PXN mRNA was measured. As shown in Fig. 5e, f, PXN-AS1-L overexpression elongated the half-life of PXN mRNA, and conversely, PXN-AS1-L knockdown shortened the half-life of PXN mRNA, which suggested that PXN-AS1-L also increase PXN mRNA stability in NSCLC cells. All these data together demonstrated that PXN-AS1-L up-regulated PXN expression in NSCLC.
PXN was up-regulated in NSCLC and positively correlated with PXN-AS1-L expression

To explore whether the regulation of PXN by PXN-AS1$\mathrm{L}$ also exist in vivo, we measured PXN expression in the same 66 pairs of NSCLC tissues and adjacent noncancerous lung tissues used in Fig. 1b. The results displayed that the expression of PXN was also significantly higher in NSCLC tissues than that in adjacent noncancerous lung tissues (Fig. 6a). Furthermore, our results also displayed that the expression of PXN was further higher in bone metastases tissues than that in primary NSCLC tissues (Fig. 6b). Correlation analysis displayed that the expression level of PXN-AS1-L was positively correlated with that of PXN in these 66 NSCLC tissues (Fig. 6c), supporting the positive regulation of PXN by PXN-AS1-L. 

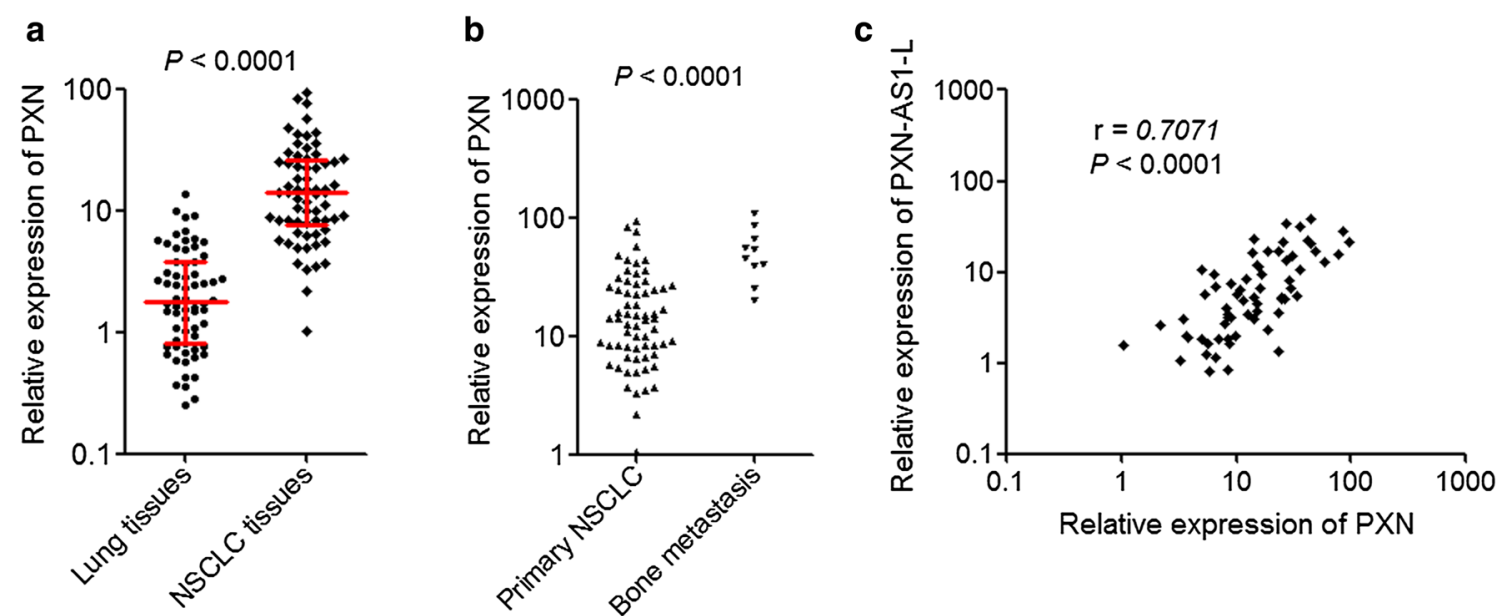

Fig. 6 PXN was up-regulated in NSCLC and positively correlated with PXN-AS1-L expression. a The expressions of PXN in 66 pairs of NSCLC tissues and adjacent noncancerous lung tissues were detected by qPCR. $P<0.0001$ by Wilcoxon signed-rank test. $\mathbf{b}$ The expressions of PXN-AS1-L in 66 primary NSCLC tissues and 10 NSCLC bone metastases tissues were detected by qPCR. $P<0.0001$ by Mann-Whitney test. c The correlation between the expression level of PXN-AS1-L and PXN was analyzed in these 66 NSCLC tissues. $r=0.7071, P<0.0001$ by Pearson correlation analysis

\section{PXN knockdown attenuated the oncogenic roles of PXN-AS1-L in NSCLC}

To explore whether the oncogenic roles of PXN-AS1$\mathrm{L}$ in NSCLC are dependent on the positive regulation of PXN, we knocked down PXN in PXN-AS1-L stably overexpressed A549 cells by transient transfection of PXN specific shRNA (Fig. 7a). Glo cell viability assays displayed that PXN knockdown attenuated the increasing of cell viability induced by PXN-AS1-L overexpression (Fig. 7b). EdU incorporation assays also displayed that PXN knockdown attenuated the pro-proliferative roles of PXN-AS1-L (Fig. 7c). TUNEL assays displayed that PXN knockdown attenuated the cell apoptosis repressive roles of PXN-AS1-L (Fig. 7d). Transwell assays displayed that PXN knockdown attenuated the increasing of cell migration induced by PXN-AS1-L overexpression (Fig. 7e). All these data together demonstrated that PXN knockdown attenuated the roles of PXN-AS1-L in promoting cell proliferation, inhibiting cell apoptosis, and promoting cell migration of NSCLC cells. These data also suggested that the oncogenic roles of PXN-AS1-L in NSCLC were at least partially dependent on the positive regulation of PXN.

\section{Discussion}

LncRNA PXN-AS1-L has 863 nucleotides in length. The gene encoding PXN-AS1-L locates at chromosome $12 \mathrm{q} 24.23$ and is reverse complementary to $P X N$. PXN-AS1-L is recently identified to have oncogenic roles in HCC [38]. In this study, we further studied the expression, roles, and mechanisms of action of PXN-AS1-L in NSCLC.
First, we found that PXN-AS1-L is up-regulated in NSCLC cell lines compared with normal bronchial epithelial cell line. Moreover, PXN-AS1-L is further upregulated in NSCLC cell lines derived from metastatic sites (NCI-H1299 and SK-MES-1) compared with that derived from primary sites (NCI-H1975 and A549). In clinical tissues specimens, we also found that PXN-AS1$\mathrm{L}$ is up-regulated in NSCLC tissues compared with noncancerous lung tissues and is further up-regulated in NSCLC bone metastases tissues. Analyses of the correlation between PXN-AS1-L expression and clinicopathological characteristics revealed that PXN-AS1-L was positively associated with tumor size, lymph nodes metastasis, advanced TNM stages, and poor prognosis. These data confirmed that PXN-AS1-L is up-regulated in NSCLC and implied that PXN-AS1-L may be involved in the progression of NSCLC. Whether the up-regulation of PXN-AS1-L in cancer is lung and liver cancer specific or cancer-popular need further investigation. Furthermore, multi-center prospective study investigating the correlation between the expression of PXN-AS1-L and prognosis of NSCLC patients would be more significant for the application of PXN-AS1-L for clinical outcome prediction.

Second, we investigated the in vitro and in vivo roles of PXN-AS1-L in NSCLC using gain-of-function and lossof-function assays. Our results revealed that PXN-AS1-L overexpression increases NSCLC cell viability, promotes NSCLC cell proliferation, inhibits NSCLC cell apoptosis, and promotes NSCLC cell migration. Conversely, PXN-AS1-L knockdown decreases NSCLC cell viability, inhibits NSCLC cell proliferation, promotes NSCLC cell 


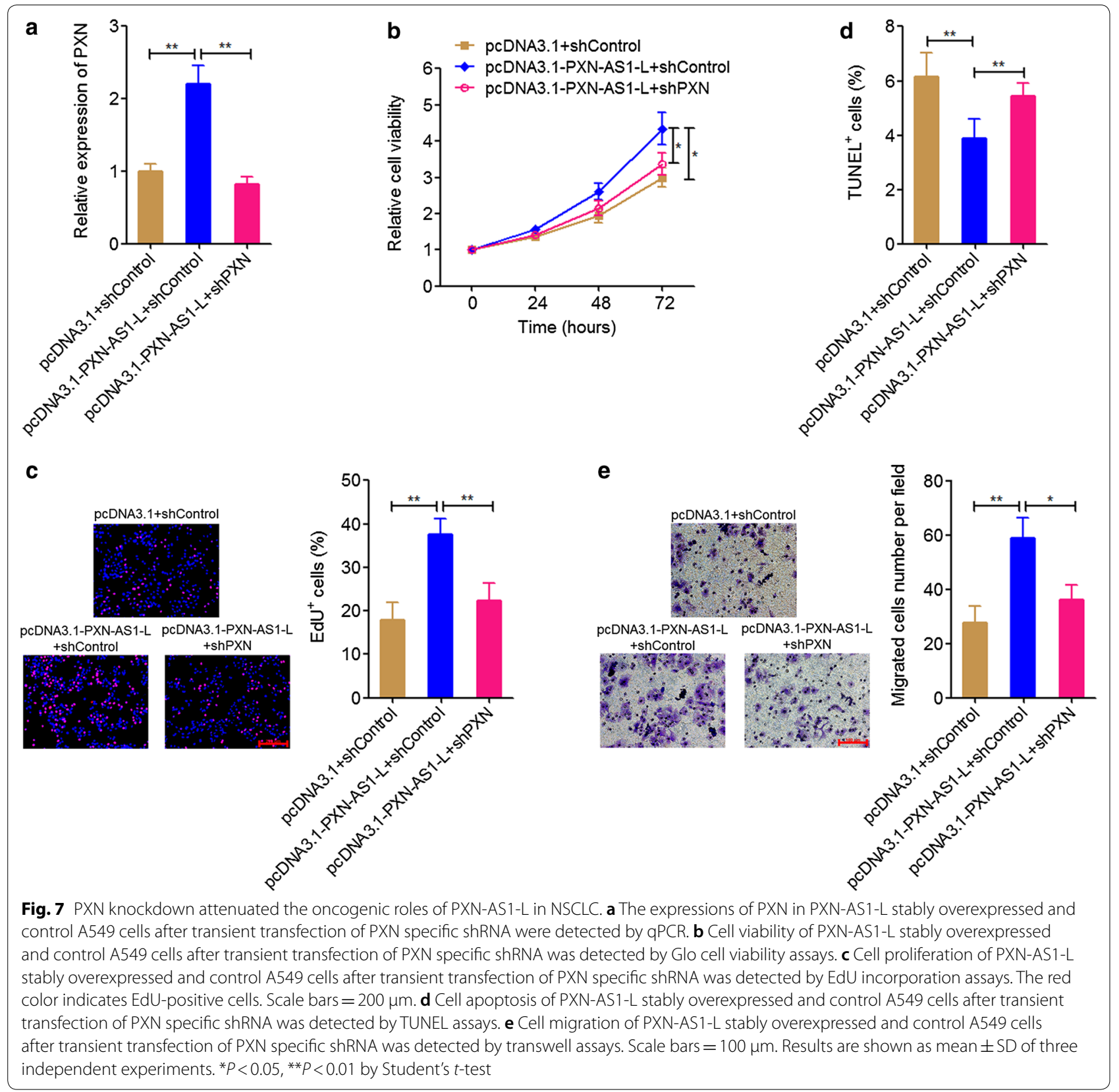

apoptosis, and represses NSCLC cell migration. Therefore, our data demonstrated that PXN-AS1-L also acts as an oncogene in NSCLC, similar to the roles of PXN-AS1$\mathrm{L}$ in HCC. Whether the oncogenic role of PXN-AS1-L is lung and liver cancer specific or cancer-popular also need further investigation. Nevertheless, the up-regulation of PXN-AS1-L and oncogenic roles of PXN-AS1-L in NSCLC suggested that PXN-AS1-L may be a potential therapeutic target for NSCLC. Increasing evidences have shown that noncoding RNAs could be targeted by chemically modified complementary oligonucleotides, which are revealed to be effective treatment in animal models and clinical trials involving humans [42, 43]. Therefore, developing chemically modified complementary oligonucleotides targeting PXN-AS1-L would be potential therapeutic strategy for NSCLC.

The focal adhesion protein PXN mediates critical signal transduction and plays important roles in cell survival and migration $[44,45]$. Due to the reverse complementation between $P X N-A S 1-L$ and $P X N$, we investigated whether PXN-AS1-L regulates PXN and whether PXN is the mediator of the oncogenic roles of PXN-AS1-L in 
NSCLC. In this study, we found that PXN-AS1-L up-regulated PXN expression. Similar to the expression pattern of PXN-AS1-L in NSCLC, PXN is also up-regulated in NSCLC tissues compared with noncancerous lung tissues and is further up-regulated in NSCLC bone metastases tissues. The expression of PXN-AS1-L is positively associated with that of PXN in NSCLC tissues. Furthermore, knockdown of PXN attenuated the oncogenic roles of PXN-AS1-L in NSCLC. All these data support the positive regulation of PXN by PXN-AS1-L and the importance of PXN in the oncogenic roles of PXN-AS1-L in NSCLC.

\section{Conclusions}

In conclusion, our data showed that PXN-AS1-L is upregulated in NSCLC, predicts poor outcome of NSCLC patients, and has oncogenic roles in NSCLC via upregulating PXN. Our data also implied that PXN-AS1-L may be a potential prognostic biomarker and therapeutic target for NSCLC.

\begin{abstract}
Abbreviations
NSCLC: non-small cell lung cancer; IncRNAs: long noncoding RNAs; HCC: hepatocellular carcinoma; qPCR: quantitative real-time PCR; EdU: ethynyl deoxyuridine; TUNEL: terminal deoxynucleotidyl transferase (TdT)-mediated dUTP nick end labeling; IHC: immunohistochemistry.
\end{abstract}

\section{Authors' contributions}

$X Z, W H$, and ZZ designed the study; ZZ, ZP, JC, JW, YH, and KS carried out the experiments; XZ, WH, ZZ, ZP, and JC collected and analyzed the data. XZ, $\mathrm{WH}$, and ZZ wrote the manuscript. All authors read and approved the final manuscript.

\section{Author details \\ 1 Department of Orthopaedic Surgery, the PLA General Hospital, Bei- jing 100000, China. ${ }^{2}$ Department of Radiology, Changzheng Hospital, Second Military Medical University, Shanghai 200003, China. ${ }^{3}$ Department of Radiol- ogy, Jinan Military General Hospital, Jinan 250031, Shandong, China. ${ }^{4}$ Depart- ment of Ultrasonography, General Hospital of Shenyang Military Region, Shenyang 110016, Liaoning, China. ${ }^{5}$ Department of Orthopaedic Surgery, China Medical University, Shenyang 110001, Liaoning, China. ${ }^{6}$ Department of General Surgery, Changhai Hospital, Second Military Medical University, Shanghai 200433, China.}

\section{Acknowledgements}

Not applicable.

\section{Competing interests}

The authors declare that they have no competing interests.

\section{Availability of data and materials}

The data used and analyzed during this study are available from the corresponding author on request.

\section{Consent for publication}

The authors agree for publication.

\section{Ethics approval and consent to participate}

Use of patient tissue samples and nude mice were approved by the ethics committee of the General Hospital of Chinese People's Liberation Army (Beijing, China).

\section{Funding}

This work was supported by grants from the National Natural Science Foundation of China (No. 81371911) and Beijing science and technique star foundation (No. 2010B80).

\section{Publisher's Note}

Springer Nature remains neutral with regard to jurisdictional claims in published maps and institutional affiliations.

Received: 10 August 2018 Accepted: 8 January 2019

Published online: 22 January 2019

\section{References}

1. Bray F, Ferlay J, Soerjomataram I, Siegel RL, Torre LA, Jemal A. Global cancer statistics 2018: GLOBOCAN estimates of incidence and mortality worldwide for 36 cancers in 185 countries. CA Cancer J Clin. 2018. https:// doi.org/10.3322/caac.21492.

2. Chen Z, Fillmore CM, Hammerman PS, Kim CF, Wong KK. Non-smallcell lung cancers: a heterogeneous set of diseases. Nat Rev Cancer. 2014;14:535-46.

3. Reck M, Heigener DF, MokT, Soria JC, Rabe KF. Management of non-smallcell lung cancer: recent developments. Lancet. 2013;382:709-19.

4. Sekimoto N, Suzuki Y, Sugano S. Decreased KPNB1 expression is induced by PLK1 inhibition and leads to apoptosis in lung adenocarcinoma. J Cancer. 2017;8:4125-40.

5. Samulin Erdem J, Skaug V, Haugen A, Zienolddiny S. Loss of MKK3 and MK2 copy numbers in non-small cell lung cancer. J Cancer. 2016;7:512-5.

6. Boldrini L, Giordano M, Niccoli C, Melfi F, Lucchi M, Mussi A, Fontanini G. Role of microRNA-33a in regulating the expression of PD-1 in lung adenocarcinoma. Cancer Cell Int. 2017;17:105.

7. Iyer MK, Niknafs YS, Malik R, Singhal U, Sahu A, Hosono Y, Barrette TR, Prensner JR, Evans JR, Zhao S, et al. The landscape of long noncoding RNAs in the human transcriptome. Nat Genet. 2015:47:199-208.

8. Schmitt AM, Chang HY. Long noncoding RNAs in cancer pathways. Cancer Cell. 2016;29:452-63.

9. Yan X, Hu Z, Feng Y, Hu X, Yuan J, Zhao SD, Zhang Y, Yang L, Shan W, He Q, et al. Comprehensive genomic characterization of long non-coding RNAs across human cancers. Cancer Cell. 2015;28:529-40.

10. Yuan JH, Yang F, Wang F, Ma JZ, Guo YJ, Tao QF, Liu F, Pan W, Wang TT, Zhou CC, et al. A long noncoding RNA activated by TGF-beta promotes the invasion-metastasis cascade in hepatocellular carcinoma. Cancer Cell. 2014;25:666-81.

11. Battaglin F, Naseem M, Puccini A, Lenz HJ. Molecular biomarkers in gastro-esophageal cancer: recent developments, current trends and future directions. Cancer Cell Int. 2018;18:99.

12. Ivanovic RF, Viana NI, Morais DR, Silva IA, Leite KR, Pontes-Junior J, Inoue G, Nahas WC, Srougi M, Reis ST. miR-29b enhances prostate cancer cell invasion independently of MMP-2 expression. Cancer Cell Int. 2018;18:18.

13. Ponting $C P$, Oliver PL, Reik W. Evolution and functions of long noncoding RNAs. Cell. 2009;136:629-41.

14. Fatica A, Bozzoni I. Long non-coding RNAs: new players in cell differentiation and development. Nat Rev Genet. 2014;15:7-21.

15. Flintoft L. Non-coding RNA: structure and function for IncRNAs. Nat Rev Genet. 2013:14:598

16. Liu X, Xiao ZD, Han L, Zhang J, Lee SW, Wang W, Lee H, Zhuang L, Chen J, Lin HK, et al. LncRNA NBR2 engages a metabolic checkpoint by regulating AMPK under energy stress. Nat Cell Biol. 2016;18:431-42.

17. Batista PJ, Chang HY. Long noncoding RNAs: cellular address codes in development and disease. Cell. 2013;152:1298-307.

18. Xu D, Yang F, Yuan JH, Zhang L, Bi HS, Zhou CC, Liu F, Wang F, Sun SH. Long noncoding RNAs associated with liver regeneration 1 accelerates hepatocyte proliferation during liver regeneration by activating Wnt/ beta-catenin signaling. Hepatology. 2013;58:739-51.

19. Zhang C, Yuan J, Hu H, Chen W, Liu M, Zhang J, Sun S, Guo Z. Long noncoding RNA CHCHD4P4 promotes epithelial-mesenchymal transition and inhibits cell proliferation in calcium oxalate-induced kidney damage. Braz J Med Biol Res. 2017;51:e6536. 
20. Lin A, Hu Q, Li C, Xing Z, Ma G, Wang C, Li J, Ye Y, Yao J, Liang K, et al. The LINK-A IncRNA interacts with Ptdlns $(3,4,5) \mathrm{P3}$ to hyperactivate AKT and confer resistance to AKT inhibitors. Nat Cell Biol. 2017;19:238-51.

21. Lin A, Li C, Xing Z, Hu Q, Liang K, Han L, Wang C, Hawke DH, Wang S, Zhang $Y$, et al. The LINK-A IncRNA activates normoxic HIF1alpha signalling in triple-negative breast cancer. Nat Cell Biol. 2016;18:213-24.

22. Zhu XT, Yuan JH, Zhu TT, Li YY, Cheng XY. Long noncoding RNA glypican 3 (GPC3) antisense transcript 1 promotes hepatocellular carcinoma progression via epigenetically activating GPC3. FEBS J. 2016:283:3739-54.

23. Li JK, Chen C, Liu JY, Shi JZ, Liu SP, Liu B, Wu DS, Fang ZY, Bao Y, Jiang MM, et al. Long noncoding RNA MRCCAT1 promotes metastasis of clear cell renal cell carcinoma via inhibiting NPR3 and activating p38-MAPK signaling. Mol Cancer. 2017;16:111.

24. Parasramka M, Yan IK, Wang X, Nguyen P, Matsuda A, Maji S, Foye C, Asmann Y, Patel T. BAP1 dependent expression of long non-coding RNA NEAT-1 contributes to sensitivity to gemcitabine in cholangiocarcinoma. Mol Cancer. 2017;16:22.

25. Fernando TR, Contreras JR, Zampini M, Rodriguez-Malave NI, Alberti MO, Anguiano J, Tran TM, Palanichamy JK, Gajeton J, Ung NM, et al. The IncRNA CASC15 regulates SOX 4 expression in RUNX1-rearranged acute leukemia. Mol Cancer. 2017;16:126.

26. Mizrahi I, Mazeh H, Grinbaum R, Beglaibter N, Wilschanski M, Pavlov V, Adileh M, Stojadinovic A, Avital I, Gure AO, et al. Colon cancer associated transcript-1 (CCAT1) expression in adenocarcinoma of the stomach. J Cancer. 2015;6:105-10.

27. Zhang L, Yang F, Yuan JH, Yuan SX, Zhou WP, Huo XS, Xu D, Bi HS, Wang F, Sun SH. Epigenetic activation of the MiR-200 family contributes to H19-mediated metastasis suppression in hepatocellular carcinoma. Carcinogenesis. 2013;34:577-86.

28. Hosseini ES, Meryet-Figuiere M, Sabzalipoor H, Kashani HH, Nikzad H, Asemi Z. Dysregulated expression of long noncoding RNAs in gynecologic cancers. Mol Cancer. 2017;16:107.

29. Gutschner T, Hammerle M, Eissmann M, Hsu J, Kim Y, Hung G, Revenko A, Arun G, Stentrup M, Gross M, et al. The noncoding RNA MALAT1 is a critical regulator of the metastasis phenotype of lung cancer cells. Cancer Res. 2013;73:1180-9.

30. Wan L, Sun M, Liu GJ, Wei CC, Zhang EB, Kong R, Xu TP, Huang MD, Wang ZX. Long noncoding RNA PVT1 promotes non-small cell lung cancer cell proliferation through epigenetically regulating LATS2 expression. Mol Cancer Ther. 2016;15:1082-94.

31. Sun M, Liu XH, Wang KM, Nie FQ, Kong R, Yang JS, Xia R, XuTP, Jin FY, Liu ZJ, et al. Downregulation of BRAF activated non-coding RNA is associated with poor prognosis for non-small cell lung cancer and promotes metastasis by affecting epithelial-mesenchymal transition. Mol Cancer. 2014;13:68.
32. Su W, Feng $S$, Chen $X$, Yang X, Mao R, Guo C, Wang Z, Thomas DG, Lin J, Reddy RM, et al. Silencing of long non-coding RNA MIR22HG triggers cell survival/death signaling via oncogenes YBX1, MET, and p21 in lung cancer. Cancer Res. 2018;78:3207-19.

33. Mao C, Wang X, Liu Y, Wang M, Yan B, Jiang Y, Shi Y, Shen Y, Liu X, Liai W, et al. A G3BP1-interacting IncRNA promotes ferroptosis and apoptosis in cancer via nuclear sequestration of p53. Cancer Res. 2018;78:3484-96.

34. Yuan S, Liu Q, Hu Z, Zhou Z, Wang G, Li C, Xie W, Meng G, Xiang Y, Wu N, et al. Long non-coding RNA MUC5B-AS1 promotes metastasis through mutually regulating MUC5B expression in lung adenocarcinoma. Cell Death Dis. 2018;9:450.

35. Li K, Sun D, Gou Q, Ke X, Gong Y, Zuo Y, Zhou JK, Guo C, Xia Z, Liu L, et al. Long non-coding RNA linc00460 promotes epithelial-mesenchymal transition and cell migration in lung cancer cells. Cancer Lett. 2018;420:80-90.

36. Chen Z, Li JL, Lin S, Cao C, Gimbrone NT, Yang R, Fu DA, Carper MB, Haura EB, Schabath MB, et al. CAMP/CREB-regulated LINC00473 marks LKB1-inactivated lung cancer and mediates tumor growth. J Clin Invest. 2016:126:2267-79.

37. White NM, Cabanski CR, Silva-Fisher JM, Dang HX, Govindan R, Maher CA. Transcriptome sequencing reveals altered long intergenic non-coding RNAs in lung cancer. Genome Biol. 2014;15:429.

38. Yuan JH, Liu XN, Wang TT, Pan W, Tao QF, Zhou WP, Wang F, Sun SH. The MBNL3 splicing factor promotes hepatocellular carcinoma by increasing PXN expression through the alternative splicing of IncRNA-PXN-AS1. Nat Cell Biol. 2017;19:820-32.

39. Jiang H, Zhang H, Hu X, Li W. Knockdown of long non-coding RNA XIST inhibits cell viability and invasion by regulating miR-137/PXN axis in nonsmall cell lung cancer. Int J Biol Macromol. 2018;111:623-31.

40. Wu DW, Chen $\mathrm{CY}$, Chu CL, Lee H. Paxillin confers resistance to tyrosine kinase inhibitors in EGFR-mutant lung cancers via modulating BIM and Mcl-1 protein stability. Oncogene. 2016;35:621-30.

41. Bi Y, Han Y, Bi H, Gao F, Wang X. miR-137 impairs the proliferative and migratory capacity of human non-small cell lung cancer cells by targeting paxillin. Hum Cell. 2014;27:95-102.

42. Rupaimoole R, Slack FJ. MicroRNA therapeutics: towards a new era for the management of cancer and other diseases. Nat Rev Drug Discov. 2017:16:203-22.

43. Slack FJ. Tackling tumors with small RNAs derived from transfer RNA. N Engl J Med. 2018;378:1842-3.

44. Huang C, Rajfur Z, Borchers C, Schaller MD, Jacobson K. JNK phosphorylates paxillin and regulates cell migration. Nature. 2003;424:219-23.

45. Liu S, Thomas SM, Woodside DG, Rose DM, Kiosses WB, Pfaff M, Ginsberg MH. Binding of paxillin to alpha4 integrins modifies integrin-dependent biological responses. Nature. 1999;402:676-81.
Ready to submit your research? Choose BMC and benefit from:

- fast, convenient online submission

- thorough peer review by experienced researchers in your field

- rapid publication on acceptance

- support for research data, including large and complex data types

- gold Open Access which fosters wider collaboration and increased citations

- maximum visibility for your research: over $100 \mathrm{M}$ website views per year

At BMC, research is always in progress.

Learn more biomedcentral.com/submissions 